\title{
Carbohydrate-derived fulvic acid wellness drink: its tolerability, safety and effect on disease markers in pre-ART HIV-1 positive subjects
}

\author{
ME Botes ${ }^{a}$, IS Giladab ${ }^{b}$ JR Snymanc* and JPL Labuschagne ${ }^{d}$ (DD \\ ${ }^{a}$ Department of Internal Medicine, University of Pretoria and Private Practitioner, Muelmed Hospital, Pretoria Private practice, Pretoria, South Africa \\ bPrivate practice, Mumbai, India \\ ${ }^{c}$ Consultant Fulhold (Pty) Ltd, Somerset West, South Africa \\ 'Private Statistician, Cape Town, South Africa \\ *Corresponding author, email:jacques@dermav.co.za

Background: Many people take wellness drinks on a daily basis to enhance physical and emotional well-being. The safety and/ or efficacy of these are not always known or even established in the populations likely to consume these. The safety, tolerability and clinical impact of CHD-FA in a formulated wellness drink (F0210) (a pure and novel fulvic acid) were researched in a pre-ART HIV-1 positive population in India an area where patients are known to use organic acids such as fulvic acids (Shilajit) for health enhancement.

Methods: This double-blind, placebo-controlled, parallel study recruited 332 patients ( $n=166$ on active; $n=166$ on placebo). The study outcomes recorded safety and tolerability data, as well as the time to ART and/or the time to a decrease in CD4 count of $100 \mathrm{cells} / \mathrm{mm}^{3}$ for subjects in each treatment group. Change in immune status is an important clinical endpoint. The secondary outcomes were CD4 count, HIV-1 viral load changes and quality of life (the latter as a further proxy for tolerability).

Results: The only notable side effect of the active medication was gastrointestinal intolerance such as diarrhoea, nausea and vomiting, which were more frequently experienced compared with placebo. The study was terminated before full recruitment due to regulatory changes in India and at the time of study termination there were too few clinical study endpoints reached to demonstrate any clinical difference between active and placebo treatments (no difference in hazard of experiencing an event (reaching indication for ART) between groups; $p=0.5724$ ). An interesting trend was that patients' CD4 counts in the study demonstrated a slower than anticipated decline compared with trends recorded in the literature for natural progression of disease.

Conclusion: The CHD FA wellness drink is well tolerated in an ART-naive study population and does not negatively affect the disease-specific parameters and hence does not adversely affect the natural progression of the HIV-1 disease or patients' general health. Further exploration of fulvic acids is therefore warranted.

Keywords: Carbohydrate derived fulvic acid (CHD-FA), HIV-1 infection, pre-ART, safety and tolerability, wellness

\section{Introduction}

Many people suffering HIV infection take wellness supplements on a daily basis to enhance physical and emotional well-being or to treat specific self-diagnosed entities. The use varies and ranges from $10 \%$ to $100 \%$ of respondents in studies. ${ }^{1,2}$ The safety and/or efficacy of these are not always known or even established in the populations likely to consume these. ${ }^{1,2} \mathrm{~A}$ novel carbohydrate derived fulvic acid (CHD-FA) based wellness drink (F0210) has extensive in-vitro and in-vivo safety data in healthy adult populations, but has only observational safety data in vulnerable patient settings such as persons with HIV-1 infections. ${ }^{3-5}$ In both acute and sub-acute dosing studies CHD-FA was found to be safe with no clinically meaningful changes in biochemistry and physical findings. ${ }^{5}$ Other humic acid derivatives (oxifulvic acid and oxihumate) also demonstrated some favourable outcomes in small clinical studies demonstrating anti-inflammatory effects or improved well-being in participants with atopy and in patients with HIV-1 respectively. ${ }^{6,7}$

Organic acids have been part of mankind's self-medication over the ages and products containing humic and/or fulvic acids such as brown coal and coal derivatives (oxifulvic and oxihumic acid) ${ }^{6,7}$ and Shilajit ${ }^{8}$ are well known for their use as wellness enhancers and have been tested for their efficacy and safety in various diseases.
Fulvic acid is a humic acid derivative ${ }^{8-10}$ known for its use as a complementary self-medication in various cultural settings. Humic Acids, and in particular Fulvic Acid, have demonstrated significant in-vitro and in-vivo anti-inflammatory properties. ${ }^{3,5,6,8}$ It is postulated that these properties may also be responsible for its purported benefits in the HIV-1 population taking CHD-FA in a formulated wellness drink. Untreated HIV infection causes persistent immune activation and inflammation, leading to a host of disease-progressive and age-related diseases. It is known that anti-inflammatory glucocorticosteroids may reduce the viral load in HIV-1 patients purely by reducing immune competent cell turnover and proliferation. ${ }^{11}$ CHD-FA results in an anti-inflammatory effect without affecting the same pathways as corticosteroids. ${ }^{3}$

In an open, non-interventional, observational, cohort study the effects of the CHD-FA containing wellness drink on the healthrelated outcomes in HIV-1 patients who used this over-thecounter product for self-medication were examined. The main outcome of this small observation study was safety; CHD-FA did not negatively impact on either the viral load or CD4 counts of the volunteers using the CHD-FA containing product. ${ }^{4}$

Antiretroviral therapy (ART) remains the mainstay of treatment against the human immuno-deficiency virus. It is now accepted that patients need to start earlier with ART. CD4 count below 200 cells $/ \mathrm{mm}^{3}$ was initially the starting target level, but that has 
changed over a short time span and the norm now can be as high as 500 cells $/ \mathrm{mm}^{3}$ depending on the geographic region and the patient's ability to fund therapy (self-funded or funded by third parties such as governments). ${ }^{12,13}$

Preserving patients' health in this pre-ART phase through safe self-care and reducing the need to commence ART would impact significantly on the treatment burden of the pandemic. The preART phase is defined as the period of time before ART is started. ${ }^{12-16}$ It is especially during this phase that HIV-1 positive patients often self-medicate by using vitamins or 'tonics' to enhance well-being. ${ }^{1,2}$ It is recognised that many patients use self-medication products because it is often easier, more cost- or time-efficient or they believe their situation does not merit a visit to a clinician. ${ }^{17,18}$ There is evidence that patients can and do practise self-medication responsibly, respect non-prescription medicines and do read package inserts. It was stated by the World Health Organization (WHO) ${ }^{18}$ that there is general recognition that self-care is undoubtedly the primary resource of any healthcare system.

The global HIV prevalence is $0.8 \%$. The vast majority of these infected individuals live in low- and middle- income countries. India ranks third in the world with regard to HIV prevalence $0.3 \%$ [2013]). This translates into 2.1 million people living with HIV. Fortunately, the epidemic is slowing down in India with a $38 \%$ decline in AIDS-related deaths between 2005 and 2013, with only 130000 new HIV infections in 2013. ${ }^{19}$ Fulvic acid forms part of the organic acids often used by patients on the Indian subcontinent as wellness enhancers or as disease treatment and is often imbibed as Shilajit. ${ }^{8}$

The aim of this study was therefore to demonstrate the safety, tolerability and clinical effect of fulvic acid in the form of CHD-FA in a formulated wellness drink on determinants of health and disease progression in a population of pre-ART, HIV-1 positive individuals.

\section{Materials and methods}

\section{Study population}

This prospective, multicentre, parallel, double-blind, placebocontrolled study recruited and enrolled pre-ART, HIV-1 positive patients in India who met the inclusion criteria and were not ineligible due to any exclusion criteria.

Subjects had to be confirmed HIV-1 positive males or females aged 18 years or older who were healthy or presented at most with WHO stage 1 or 2 disease and a CD4 count between 250 cells $/ \mathrm{mm}^{3}$ and 550 cells $/ \mathrm{mm}^{3}$. India's country-specific guidelines determined the CD4 count for inclusion. The CD4 inclusion threshold was 250 cells $/ \mathrm{mm}^{3}$ at study initiation, but this was updated during the study to accommodate the change in the country-specific guidelines to 350 cells $/ \mathrm{mm}^{3,14,15}$ Subjects had to be ART-naive. Females of childbearing potential were eligible only if they were not sexually active or if they followed a medically accepted contraceptive method. The liver enzyme markers of the subject had to be below twice the upper limit of the normal range; subjects had to have normal kidney function as well as a haemoglobin above $8 \mathrm{~g} / \mathrm{dl}$.

Pregnant or breastfeeding women were excluded, as well as patients with systemic uncontrolled diseases, malignancies and chronic diarrhoea, which may lead to malabsorption. The use of herbal self-administered medicine was also an exclusion criterion for recruitment.

All participants received routine care according to local guidelines and practice-specific criteria.

\section{Ethics}

Participants provided written informed consent before enrolment. The study protocols were approved by the institutions' specific research ethics committees. These included Mahavir Hospital and Research Center, Axon Hospitals, and Clinicom for private practice sites, respectively, within their jurisdictions taking part in the study. Participants were followed up as for their routine visits at their private clinics where they were patients and received no remuneration other than out-of-pocket expenses for taking part in the study. The study sponsor (Fulhold (Pty), Somerset West, South Africa) carried sufficient insurance for the study participants as required by the IRB (HDFC ERGO_clinical trial-certificate for Endorsement to Policy no. 299920022504970000). This study was conducted according to good clinical practice (GCP) guidelines and the World Medical Association Declaration of Helsinki Ethical Principles for Medical Research Involving Human Subjects (2008).

\section{Study outcomes}

\section{The study as a whole had predefined early stopping criteria:}

- First, if $40 \%$ of the subjects initiated ART and/or experienced a decrease in CD4 count of 100 cells $/ \mathrm{mm}^{3}$ or more from baseline (disease deterioration). This was seen as a significant and clinically meaningful change in immune status warranting ARV treatment.

- Premature discontinuation of the study due to adverse events being perceived as more than placebo as adjudicated by the independent data safety monitoring board (DSAMB). This detrimental effect of the active treatment was defined as: If, after at least $50 \%$ of the subjects spent at least 30 weeks under observation, there was a statistically significant detrimental effect of $\mathrm{FH} 0210$ compared with the placebo group.

- If a large positive effect was observed, if it could be shown that a reduction in initiation of ART of at least 30\% (as per above definition).

An individual subject completed the study when one of the following events occurred: The study was stopped for one of the reasons given above; the treating clinician decided to initiate ART for the subject and/or the subject experienced a decrease in CD4 count of 100 cells $/ \mathrm{mm}^{3}$ or more, and/or the subject withdrew from the study for whatever reason.

\section{The outcomes were defined as follows:}

- The primary outcome of the study was to determine and compare the time to ART and/or the time to a decrease in CD4 count of 100 cells $/ \mathrm{mm}^{3}$ for subjects in each treatment group (impact on disease).

- The secondary outcomes were:

- CD4 counts and viral loads;

- Change in Quality of Life RAND SF36;

- Safety outcomes of the product in terms of organ function side effect profile. 


\section{Active substance}

Humic substances are present wherever organic matter is decomposing. ${ }^{8-10}$ Humic substances can be defined as:'a general category of naturally occurring, biogenic, heterogeneous organic substances that can generally be characterized as being yellow to black in colour and of high molecular weight'. Humic substances can be subdivided into humic acid (HA), fulvic acid (FA) and humin. The average molecular weight of humic substances may range from 500 to $5000 \mathrm{Da}$ for FA and 3000 to 1 000000 Da for HA. ${ }^{9}$ These substances are characterised on the basis of their solubility in water as a function of $\mathrm{pH}$. FA is the fraction that is soluble in water under all $\mathrm{pH}$ conditions. In general, FA is also lower in molecular size and weight and lower in colour intensity than the HA. The novel medicinal properties and applications of HA and FA have been researched over many decades for various applications. ${ }^{8,9}$

Fulvic acids, such as Shilajit, that are mined from natural sources contain high concentrations of heavy metals, including mercury, aluminium, chromium, lead and cadmium and are not consistent from batch to batch with regard to efficacy and quality and are therefore not appropriate for use in medical, pharmaceutical and cosmetic preparations. Carbohydrate sources such as glucose, sucrose, fructose, starches and cellulose have successfully been treated by wet oxidation to produce a CHD-FA composition that is now internationally patented because of its uniqueness (SA Patent 2001/2419). This CHD-FA (of unique molecular weight and purity) is suitable for use in medical, pharmaceutical and cosmetic preparations as it contains only a negligible, if any, amount of heavy metals and is consistent from batch to batch. ${ }^{9}$

The investigational product of the present study (F0210) is CHDFA in a known formulated wellness drink. The usual daily dose is $15 \mathrm{ml} /$ dose which contains $14.68 \mathrm{ml}$ CHD-FA with $4-5.5 \%$ active matter, $300 \mu \mathrm{g}$ selenium AAC, $257 \mathrm{mg}$ L-glutamine and $0.03 \mathrm{ml}$ raspberry flavourant. In order to ensure the best possible compliance in the present study the former was made up to $50 \mathrm{ml}$ in water and packed in dosages of $50 \mathrm{ml} /$ dose as a premixed solution taken three times daily. The dose was not challenged in the study but taken from the manufacturer's label. There is

Table 1: Patient demographics at screening and last available visit therefore no dose justification other than the use of commercialised product labelling.

\section{Placebo}

The placebo drink was formulated to also have an acidic taste and was the same colour as the active version. The placebo wellness drink was manufactured by the manufacturers of CHDFA (FulviMed (Pty), Stellenbosch, South Africa), according to good manufacturing practice (GMP) principles.

\section{Sample size}

This study was designed to include a total of 460 HIV-1 positive, pre-ART, ART-naive subjects to power the study for 184 events as endpoint. Such an endpoint event was defined earlier.

The CD4 count typically declines over time as HIV-1 infection progresses and should be monitored 3-4 monthly. ${ }^{14,15}$ Based on published data it was calculated that the mean rate of decrease in CD4 count is 70 cells $/ \mathrm{mm}^{3}$ per year for HIV-1 positive patients. ${ }^{20}$ This then formed the basis for the study size calculation and statistical plan design. Assuming that the rate of decrease in CD4 count is between 70 and 90 cells $/ \mathrm{mm}^{3}$ per year, it was anticipated that the study period would be no longer than 24 months (96 weeks).

\section{Randomisation and treatment groups}

The planned study population was divided into two groups, with 230 anticipated subjects in the placebo and active groups respectively.

For each centre two site-specific randomisation lists were constructed. The study was balanced and a block approach with a block size of eight was used. Blinding was ensured as allocation numbers was generated centrally and dispatched to the treatment centres based on recruitment of subjects. Emergency un-blinding was possible as coding was kept at local CRO level with someone reachable $24 \mathrm{~h}$ per day every day.

\section{Study process and visits}

Visit 1 (Day -5 TO -14) was for screening and informed consent; visit 2 was the randomisation visit conducted within 14 days of

\begin{tabular}{|c|c|c|c|c|}
\hline \multirow[b]{2}{*}{ Demographic data at inclusion } & & \multicolumn{3}{|c|}{ Inclusion } \\
\hline & & \multicolumn{2}{|l|}{ Placebo } & Active \\
\hline Age (mean) & & \multicolumn{2}{|l|}{39.2} & 36.5 \\
\hline \multicolumn{5}{|l|}{ Gender: } \\
\hline Male $(n)$ & & \multicolumn{2}{|l|}{90} & 89 \\
\hline Female $(n)$ & & \multicolumn{2}{|l|}{76} & 77 \\
\hline HIV-1 (mean time since diagnosis in year & & \multicolumn{2}{|l|}{2.56} & 2.11 \\
\hline $\begin{array}{l}\text { Mean change in parameters over } \\
\text { time }\end{array}$ & Placebo at inclusion & Active at inclusion & Placebo last available visit* & Active last available visit* \\
\hline CD4 (mean cells $/ \mathrm{mm}^{3}$ ) & 419 & 413 & 447 & 449 \\
\hline Viral load (mean log) & 3.62 & 3.59 & 3.56 & 3.33 \\
\hline Creatinine (mean) & 0.917 & 0.847 & 0.88 & 0.86 \\
\hline GGT (mean mmol/l) & 35.2 & 33.3 & $42.23(37.71 \dagger)$ & 36.19 \\
\hline SGOT (mean mmol/l) & 28.8 & 28.7 & 31.2 & 28.9 \\
\hline Haemoglobin (mean mg/dl) & 13.1 & 13.1 & 13.1 & 13.0 \\
\hline
\end{tabular}

Note: From this table it is clear that there was no significant difference between the two arms at study initiation and study end.

*The last available visit is the last visit for the patient before the study termination date that was not the screening visit and that had a valid measurement for the parameter.

${ }^{\dagger}$ One patient had a very high GGT value of 674. The value in parentheses shows the average when this outlier is removed. 


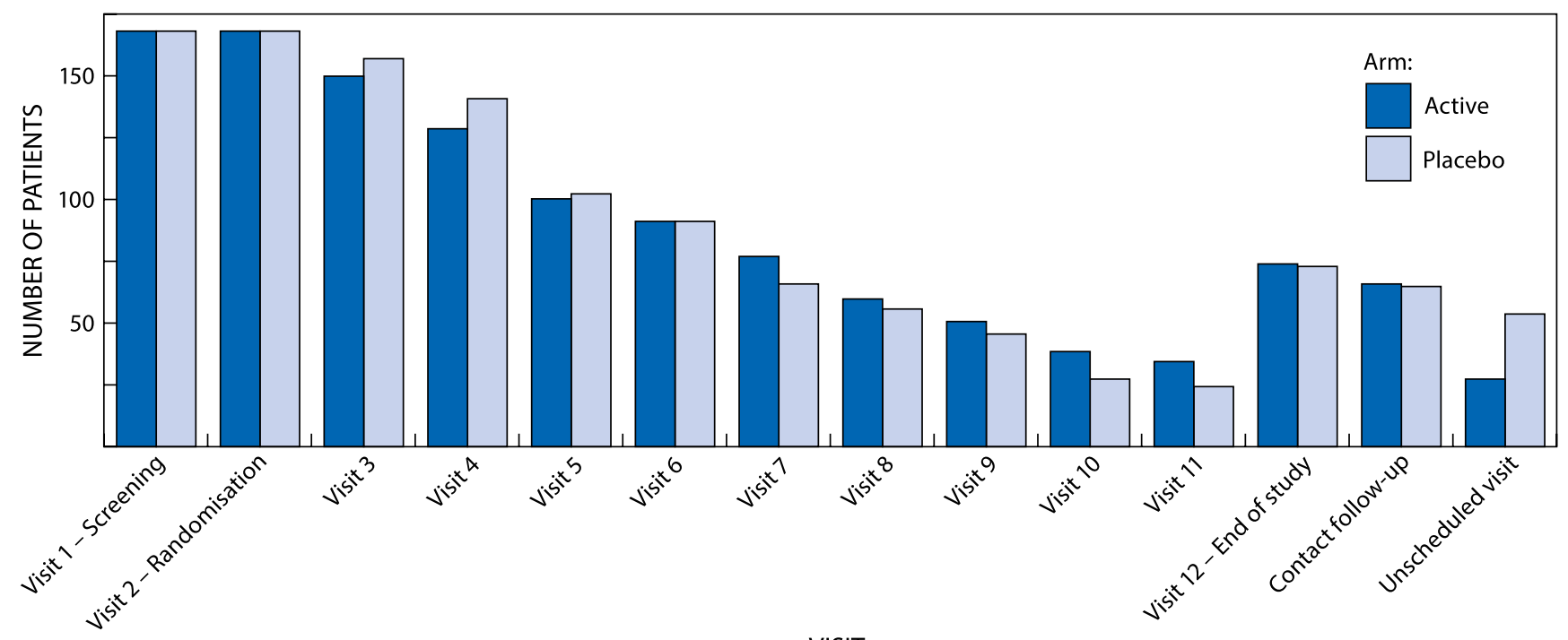

VISIT

Figure 1: Total number of patients who went for each visit prior to study termination.

Note: The study is highly censored after the 6th and 7th visits due to early termination of the study. The graph show patients per visit as some patients skipped some visits and others stopped before final visit or study completion. The report is however on all recruited patients (ITT analyses). Data is of patient who had at least two visits.

the screening visit. Visit 3 followed 4 weeks later ( +5 days) to ensure patient understanding and compliance. Thereafter the visits were spaced 12 weeks apart till study end as this was the normal practice for follow-up periods. It was planned that the average patient would be on the study for 12 months. The end of study visit was conducted at the end of the study period or earlier when a patient-specific endpoint was reached. During each visit, each patient underwent a complete physical examination and compulsory blood sample collection to determine CD4 cell count, HIV-1 RNA plasma load (VL) as well as liver and kidney function tests and basic haematology. Visits also recorded functional health and well-being with the RAND SF36 scores, compliance and adverse event monitoring data.

\section{Unscheduled visits}

Unscheduled visits were arranged with subjects as dictated by clinical need.

\section{Verification of compliance}

Compliance was achieved when a subject had used $80 \%$ of his/ her medication for a period of at least 24 out of 30 days (80\%) each month. A compliance rate of between $60 \%$ and $80 \%$ was noted as reduced compliance, whereas a compliance rate of less than $60 \%$ was deemed non-compliant. If a subject was noncompliant for more than two consecutive visits, the subject had to be withdrawn. Compliance was assessed by the clinician at every visit by collecting all returned medication and weighing the unused and returned containers in a central laboratory for verification.

\section{Results}

Of the planned 460 patients, only 332 were recruited over a period of 36 months. They were evenly distributed resulting in 166 per treatment group as the study was stopped prematurely due to regulation changes in India. At baseline, the median age was 36.5 in the active group vs. 39.2 in the placebo group. The male:female ratio was $89: 77$ vs. $90: 76$ in the active and placebo groups respectively. The baseline average CD4 counts were 413 in the active vs. 419 in the placebo group. Table 1 provides more detail of patient demographics. Baseline characteristics were comparable for both treatment arms. None of the differences in ratios as recruited per arm demonstrated any significant or clinically meaningful difference. Only five patients missed more than one study visit with the maximum being two visits skipped (Figure 1 depicts study visits per study arm).

There was no impact detected on the patients' disease status, i.e. prolongation of time to ART or slowing of disease deterioration or improvements in CD4 counts and/or viral loads (no statistical differences detected between placebo and active treatment groups for all parameters assessed during the study period). The primary safety objective was reached, namely no deterioration of HIV-1 disease markers or any impact on organ function over time. An interesting trend to note was that the recruited patients' CD4 counts in the study deteriorated significantly slower than recorded in the literature for both active and placebo treated patients (see Figure 2).

The study was terminated early due to the DCGI (Drug Controller General of India) in India changing a regulation such that no clinical trial, within India, based on nutraceutical products was allowed unless prior approval was given by the DCGI and since this study was initiated prior to this regulation change the study had to be stopped. DCGI approval was not a requirement at start of study.

As can be seen from Tables 2 and 3 only a few patients per treatment arm reached the study endpoint due to either experiencing a decline of 100 cells $/ \mathrm{mm}^{3}$ or more in CD4 count or receiving ART or becoming ART eligible according to the treating clinician. There were no differences detected between sites (see Table 3).

There was also no change in either physical or mental well-being as determined by the RAND SF36 scores. 


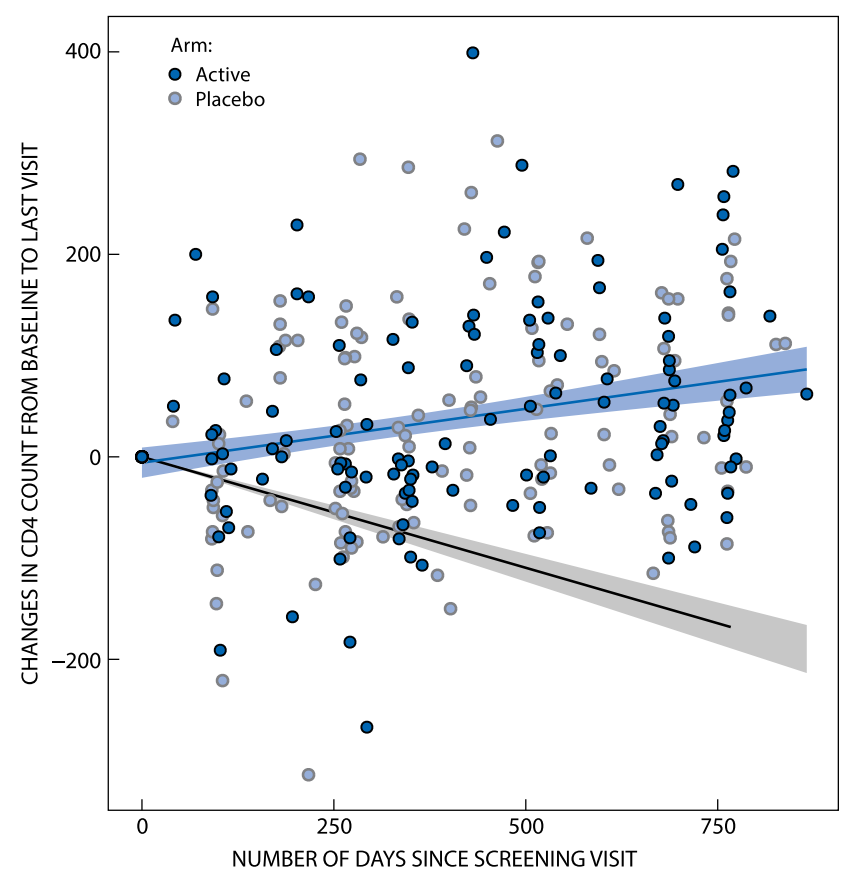

Figure 2: Summary of CD4 changes from baseline superimposed on anticipated decline.

Notes: The black line indicates the anticipated CD4 decline of between 70 and 90 cells per $\mathrm{mm} 3$ per year. The blue line was fitted to the observed data from the trial. The $95 \%$ confidence interval of change in CD4 count per year for the population as a whole is an increase of between 23.66 and 66.21 cell per $\mathrm{mm}^{3}$ per year.

There was a slight non-significant difference in the reported side effects due to gastrointestinal tolerance (diarrhoea) as well as headache being reported more frequently in the actively treated group (see Table 4).

The Indian Government changed its HIV-1 guidelines (NANCO) at the end of $2012^{15}$ and increased the threshold for initiation of

Table 2: Detailed discontinuation reasons per patient

\begin{tabular}{lccc}
\hline Item & Active & Placebo & Sum \\
\hline ART initiated & 4 & 3 & $7^{*}$ \\
\hline $\begin{array}{l}\text { Became ineligible** } \\
\text { CD4 250 (reached stopping criteria at level } \\
\text { of count) }\end{array}$ & 0 & 1 & 1 \\
$\begin{array}{l}\text { CD4 350 (reached stopping criteria at level } \\
\text { of count) }\end{array}$ & 5 & 2 & $7^{*}$ \\
$\begin{array}{l}\text { CD4 threshold (reached stopping criteria as } \\
\text { per rate in decline in CD4) }\end{array}$ & 2 & 5 & $7^{*}$ \\
$\begin{array}{l}\text { Completed visits } \\
\text { Consent withdrawn }\end{array}$ & 26 & 18 & 44 \\
\hline $\begin{array}{l}\text { Continuing } \\
\text { CD4 decline by 100 cells }\end{array}$ & $17^{\sharp}$ & 8 & $25^{\dagger}$ \\
\hline $\begin{array}{l}\text { Liver enzymes high } \\
\text { Lost to follow-up }\end{array}$ & 1 & 2 & 4 \\
\hline
\end{tabular}

*From this table it is obvious that only 36 events occurred.

**Patients becoming non-compliant or missing two visits in a row. ${ }^{\dagger}$ More patients withdrew consent on active treatment.

*The numbers lost to follow-up were no different in the two groups. \#Patients could with draw consent without stating a reason (mostly due to taste).
Table 3: Event summary

\begin{tabular}{lccc}
\hline Item & Active & Placebo & Total \\
\hline Total & 16 & 20 & 36 \\
Female & 7 & 13 & 20 \\
Male & 9 & 7 & 16 \\
Site 1 & 3 & 3 & $6(9 \%)$ \\
Site 2* & 5 & 3 & $8(6.3 \%)$ \\
Site 3 & 5 & 12 & $17(16.7 \%)$ \\
\hline Site 4 & 3 & 2 & $5(13.9 \%)$ \\
\hline
\end{tabular}

Note: Items in parentheses indication ratio to number of patients per site.

* Site 2 had 127 patients but lowest ratio.

Table 4: Number of patients per treatment arm that reported adverse events

\begin{tabular}{lccc}
\hline Item & Active & Placebo & Sum \\
\hline Acute nasopharyngitis (common cold) & 8 & 11 & 19 \\
\hline Myalgia & 6 & 6 & 12 \\
\hline Cough & 4 & 6 & 10 \\
\hline Fever of unknown origin & 3 & 5 & 8 \\
\hline Headache & 6 & 2 & 8 \\
\hline Diarrhoea unspecified & 5 & 2 & 7 \\
\hline Gastritis simple & 4 & 1 & 5 \\
\hline Pain in lower limb unspecified & 1 & 4 & 5 \\
\hline Nausea and vomiting & 4 & 0 & 4 \\
\hline Pruritus & 1 & 3 & 4 \\
\hline Abnormal results of liver function studies & 0 & 3 & 3 \\
\hline Anorexia & 2 & 1 & 3 \\
\hline Herpes zoster & 2 & 1 & 3 \\
\hline Lower back pain & 1 & 2 & 3 \\
\hline Weakness non-specific & 3 & 0 & 3 \\
\hline
\end{tabular}

Note: This table lists the adverse events reported where the total number was more than two. As can be seen, the gastrointestinal adverse events (diarrhoea) as well as headaches were more frequently reported in the active treatment arm; this was, however, not significantly different between the groups.

ART from a CD4 count of 250 to 350 cells $/ \mathrm{mm}^{3}$. All new patients recruited after this point were subject to the new higher threshold for inclusion, and all patients with CD4 counts below this value at the time were given the option to go onto ART immediately. This did not affect the outcome of the study.

\section{Discussion}

The main finding of this study is that the CHD-FA containing wellness drink F0210 demonstrated no detrimental effects on the progression of disease in HIV-1 infected pre-ART patients and that if anything the study trended to make patients better off than the natural described progression of disease. Organ function was maintained and no serious adverse events occurred, and most of the side effects noted were not different from placebo.

Instead of the CD4 count reducing over time as anticipated, this study demonstrated that the $95 \%$ confidence interval of the change in CD4 count per year for the population as a whole is an increase of between 23.66 and 66.21 cells per $\mathrm{mm}^{3}$ (Figure 2). This finding should be explored further in future studies. There was no significant difference from baseline within groups and also no difference between treatment arms for the outcomes 
marking disease deterioration or improvement, that is the hazard of experiencing a decline in CD4 count of 100 cells $/ \mathrm{mm}^{3}$ or reaching indications for initiating ART (CD4 count or AIDS defining disease as determined by the treating clinician).

The influence of changing the threshold for the initiation of ART (decline in CD4 count to below 350 cells $/ \mathrm{mm}^{3}$ ) by the Indian guideline committee did not influence the outcome, neither did any specific site. The only predictors of reaching threshold were the initial CD4 count and the viral load. Those with the lowest CD4 counts and highest viral loads were more likely to reach an event as defined by this study $(p=0.0076$ and $p=0.0211$, respectively).

The major limitation of the study, for demonstrating any effect on disease-specific markers, was the early termination of the study. However, sufficient data were collected to demonstrate tolerability and safety of the imbibed organic acid under investigation. There were also no early warning signs associated with the CHD-FA wellness drink with regard to observed clinical and laboratory parameters. The treatment with CHD-FA in a formulated wellness drink was demonstrated to be safe and well tolerated. It should, however, be noted that seven patients in the placebo arm had to withdraw from the study due to elevation in liver enzymes, while this did not happen in the CHD-FA arm. The patients' quality of life scores showed a minor upward trend over the duration of the study.

This study therefore provides data for the safety of a CHD-FA containing wellness drink in a particular vulnerable population, therefore also indicating that the CHD-FA is safe for use in a normal population.

\section{Conclusion}

The finding of this study has clinical relevance, as clinicians often have no control over self-medication ${ }^{1,2}$ and patients are then unwittingly exposed to the potential side effects of these which is not the case with this CHD-FA wellness drink.

Acknowledgements - The study was sponsored by Fulhold (Pty).This study was conducted under the supervision of the following Drug Safety Advisory Board: Dr Adri Kok: Independent Physician - Chairman; Prof Oppel Greeff: Head Department of Pharmacology University Pretoria; $\mathrm{Dr}$ Mariëtte Botes: Independent Physician and Principle Investigator; Dr Jaco Jurgens: Independent Physician and Clinical Researcher.

\section{Author conflict of interest declaration}

ME Botes, IS Gilada and JPL Labuschagne had no material interest in the sponsor company, but received remuneration for time spent on the study. JR Snyman is a consultant for Fulhold (Pty) and receives remuneration for consultative services.

\section{ORCID}

JPL Labuschagne (D) http://orcid.org/0000-0002-1199-2768

\section{References}

1. Bouic PJD. HIV infection and OTC supplements: do they really have an impact? CME 2003;201(2): 60-63.
2. Biudes MF, Galato D. Self-medication in patients living with HIV/AIDS little measured reality. Int J Pharm Sci Rev Res. 2014;25(2): 115-119.

3. Sabi R, Medlen CE. An investigation into the topical and systemic safety and efficacy of a new carbohydrate derived fulvic acid (CHDFA) product. Unpublished results. Data on file. Pretoria: University of Pretoria; 2008

4. Gandy JJ, Snyman JR, Medlen CE. An open observational study into the effects of oral use of Secomet $V^{\circledR}$ on health-related outcomes in patients taking the product as a so called supplement for the treatment of HIV. Unpublished results. Data on file. Pretoria: University of Pretoria; 2008.

5. Gandy JJ, Meeding JP, Snyman JR, et al. Phase 1 clinical study of the acute and sub-acute safety and proof-of-concept efficacy of carbohydrate-derived fulvic acid. Clin Pharmacol. 2012;4: 7-11.

6. Snyman JR, Dekker J, Malfeld SCK, et al. Pilot study to evaluate the safety and therapeutic efficacy of topical oxifulvic acid in atopic volunteers. Drug Dev Res 2002;57: 40-43. https://doi.org/10.1002/ (ISSN) 1098-2299

7. Botes $M E$, Dekker J, van Rensburg CEJ. Phase I trial with oral oxihumate in HIV infected patients. Drug Dev Res 2002;57: 34-39. https://doi.org/10.1002/(ISSN)1098-2299

8. Shilajit, a scientific review [cited 2007 Jan 23]. Available from: https:// examine.com/supplements/shilajit/

9. Hayes MHB et al. The search for structure: setting the scene. In: Hayes MHB, MacCarthy P, Malcom RL, Swift RS, editors. Humic substances II: in search of structure. London: Wiley; 1989.

10. Sparks DL. Environmental soil chemistry. 2nd ed. San Diego, CA: Academic Press; 2003. p. 82.

11. Cole SW. Psychosocial influences on HIV-1 disease progression: Neural, endocrine, and virologic mechanisms. Psychosom Med 2008;70(5) 562-568. https://doi.org/10.1097/PSY.0b013e3181773bbd

12. Wilkin TJ, Gulick RM. When to start antiretroviral therapy? Clin Infect Dis. 2008;47: 1580-1586. https://doi.org/10.1086/596321

13. Anglemyer A, Rutherford GW, Easterbrook PJ, et al. Early initiation of antiretroviral therapy in HIV-infected adults and adolescents: a systematic review. AIDS. 2014;28(Suppl 2): S105-S118. https://doi.org/10.1097/QAD.0000000000000232

14. National AIDS Control Organisation. Antiretroviral therapy guidelines for HIV infected adults and adolescence including post-exposure prophylaxis. New Delhi: Government of India, Ministry of Health \& Family Welfare; 2007 May. p. 136.

15. National AIDS Control Organisation. Antiretroviral therapy guidelines for HIV infected adults and adolescents. New Delhi: Government of India, Ministry of Health and Family Welfare; 2013 May.

16. The South African antiretroviral treatment guidelines. 2013. Department of Health: Republic of South Africa; 2013 March.

17. The use of essential drugs: eighth report of the WHO Expert Committee (including the revised Model List of Essential Drugs). WHO Technical Report Series 882. Geneva: World Health Organization; 1998. Available from: http://apps.who.int/medicinedocs/documents/ s16061e/s16061e.pdf

18. WHO working party on the role of the pharmacist in self-care and self-medication; 1998. Available from: http://www.smasa.cc/ standardpage.php? sitecontentid=8\&menuid=61\&title=Overview

19. UNAIDS. HIV and AIDS in India. In: Global AIDS Update 2016. 2016 [cited 2017 Nov 25]. Available from: http://www.avert.org/ professionals/hiv-around-world/asia-pacific/india

20. Flanigan TP, Imam N, Lange N, et al. Decline of CD4 lymphocyte counts from the time of seroconversion in HIV-positive women. J Women's Health 1992;1(3): 231-234. https://doi.org/10.1089/jwh.1992.1.231

Received: 10-05-2017 Accepted: 22-10-2017 